\title{
EQUACIONAMENTO DA QUALIDADE INICIAL DE SEMENTES DE MILHO E SOJA PARA APLICAÇÃO NO MODELO DE PROBIT ${ }^{1}$
}

\author{
ROBERTO SINÍCIO², MARIA CARMEN BHERING², DEBORAH DE SOUZA VIDIGAL ${ }^{3}$, \\ DENISE CUNHA FERNANDES SANTOS DIAS ${ }^{4}$
}

\begin{abstract}
RESUMO - Objetivou-se, neste trabalho, equacionar a qualidade inicial das sementes (Ki), utilizada no modelo de probit, para a previsão da longevidade de sementes de milho (Zea mays) e soja (Glycine max), em função do resultado dos testes iniciais de germinação e vigor. Onze lotes de sementes de diferentes cultivares de milho e de soja foram submetidos aos testes de germinação (TG), envelhecimento acelerado (TEA), frio sem solo (TF), para milho, e tetrazólio (TZ), para soja, e a testes de envelhecimento acelerado, com condições constantes do grau de umidade e da temperatura das sementes, para determinar o valor de Ki. Dados da literatura contendo resultados de testes de TEA e TF para milho e TEA e TZ para soja também foram usados nesta pesquisa. Conduziram-se análises de regressão linear simples e múltipla para equacionar: a) Ki em função de TG e TEA para milho e soja, b) $T E A_{m}$ (envelhecimento acelerado do milho) em função de TF; c) $T E A_{s}$ (envelhecimento acelerado da soja) em função de $T Z$. As equações obtidas foram: $K i=0,4230+0,4464 T G+0,4131$ TEA TEA $=-0,4408+1,214 T F T E A_{S}=-0,1872+0,9875$ TZ. A equação de Ki apresentou coeficiente de determinação ajustado $\left(\mathrm{r}^{2}\right)$ de 0.80 (d.p. 0.18 probit e parâmetros significantes em $\mathrm{p}<0.01, \mathrm{p}<0.02$ e p $<0.05$, respectivamente). A equação de $T E A_{m}$ apresentou $\mathrm{r}^{2}$ de 0.80 (d.p. 0.24 probit e parâmetros significantes em $\mathrm{p}<0.002$ ). A equação de $T E A_{s}$ apresentou $\mathrm{r}^{2}$ de 0.84 (d.p. 0.18 probit e parâmetros significantes em $\mathrm{p}<0.005)$. O valor de $K i$ pode ser equacionado em função do resultado dos testes iniciais de germinação e vigor para milho e soja (TEA ou TF para milho e TEA ou TZ para soja).
\end{abstract}

Termos para indexação: equação, previsão da viabilidade, Zea mays, Glycine max.

\section{EQUATING THE INITIAL QUALITY OF CORN AND SOYBEAN SEED FOR APPLICATING IN THE PROBIT MODEL}

\begin{abstract}
The objective was to equate the initial quality of the seeds (Ki), used in the probit model for predicting the longevity of corn (Zea mays) and soybean (Glycine max), as a function of the result of the initial tests of germination and accelerated ageing. Eleven seed lots of different corn and soybean cultivars were submitted to the tests of germination (TG), accelerated ageing (TEA), cold without soil (TF), for corn, and tetrazolium (TZ), for soybean, and to accelerated ageing tests, with constant conditions of moisture content and temperature of the seeds, to determine the value of Ki. Literature data with results of TEA and TF for corn and TEA and TZ for soybean were also used in this research. Simple and multiple linear regression analyses were conducted to equate: a) $\mathrm{Ki}$ as a

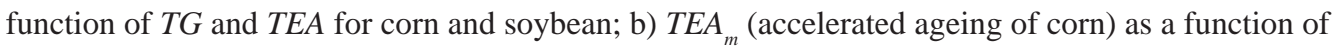
$T F$; c) $T E A_{s}$ (accelerated ageing of soybean) as a function of $T Z$. The equations obtained were: $K i=$
\end{abstract}

${ }^{1}$ Submetido em 14/04/2008. Aceito para publicação em 18/10/2008. Financiado pela FAPEMIG e SEBRAE.

${ }^{2}$ Pesquisadores do Departamento de Fitotecnia, Universidade Federal de Viçosa, 36570-000 Viçosa, MG. E-mail: rsinicio@ufv.br; mbhering@ufv. br
${ }^{3}$ Doutoranda do Departamento de Fitotecnia, Universidade Federal de Viçosa, 36570-000 Viçosa, MG. E-mail: dsvidigal@gmail.com

${ }^{4}$ Prof. Associado do Departamento de Fitotecnia, Universidade Federal de Viçosa, 36570-000 Viçosa, MG. E-mail: dcdias@ufv.br 
0,4230 + 0,4464 TG + 0,4131 TEA TEA $=-0,4408+1,214$ TF TEA $A_{S}=-0,1872+0,9875$ TZ. The Ki equation presented an adjusted determination coefficient $\left(\mathrm{r}^{2}\right)$ of 0,80 (d.p. 0,18 probit and parameters significant in $\mathrm{p}<0,01, \mathrm{p}<0,02 \mathrm{e} \mathrm{p}<0,05$, respectively). The $T E A_{m}$ equation presented $\mathrm{r}^{2}$ of 0,80 (d.p. 0,24 probit and parameters significant in $\mathrm{p}<0,002$ ). The $T E A_{s}$ equation presented $\mathrm{r}^{2}$ of 0,84 (d.p. 0,18 probit and parameters significant in $\mathrm{p}<0,005$ ). The value of $K i$ can be equated as a function of the result of the initial tests of germination and vigor for corn and soybean (TEA or TF for corn and $T E A$ or $T Z$ for soybean).

Index terms: equation, viability prediction, Zea mays, Glycine max.

\section{INTRODUÇÃO}

A previsão da perda de viabilidade de sementes armazenadas é importante tanto para a manutenção de bancos de germoplasma como para o gerenciamento da produção e armazenamento comercial de sementes. Assim, torna-se necessário disponibilizar modelos matemáticos confiáveis para predizer a longevidade das sementes armazenadas (Mead e Gray, 1999).

A necessidade de monitoramento da qualidade das sementes armazenadas é diminuída na medida em que são desenvolvidos modelos mais precisos. O monitoramento da viabilidade é a etapa que exige maior demanda de mão-deobra para a manutenção de bancos de germoplasma (Bewley e Black, 1994).

Ellis e Roberts (1980) apresentaram o seguinte modelo de probit para predizer a perda de viabilidade de sementes armazenadas:

$$
\begin{aligned}
& v=\mathrm{ki}-p / \sigma \\
& \sigma=10^{\left(K E-C W \log m-C H t-C Q l^{2}\right)} \\
& \text { em que: }
\end{aligned}
$$

$p$ = período de tempo (d);

$\sigma=$ desvio padrão da freqüência de distribuição das sementes mortas durante o período de armazenamento (d);

$m$ = grau de umidade (\%, base úmida);

$t=$ temperatura $\left({ }^{\circ} \mathrm{C}\right)$; e

$K E, C W, C H$ e $C Q=$ constantes específicas para cada espécie, independentes do genótipo e das condições de préarmazenamento.

A perda da germinação de sementes armazenadas, em função do período de armazenamento, temperatura do ambiente e grau de umidade das sementes, tem sido prevista com sucesso para um grande número de espécies e cultivares, utilizando-se o modelo de probit proposto por Ellis e Roberts
(1980). Os parâmetros KE, CW, CH e CQ deste modelo já foram determinados, por diferentes pesquisadores, para sementes de mais de 77 cultivares (mais de 48 espécies), conforme Hong et al. (1996)

O parâmetro Ki representa uma estimativa da qualidade inicial das sementes, sendo específico para cada lote de sementes, dependente dos efeitos isolados e da interação entre genótipo e condições de pré-armazenamento (Ellis e Roberts, 1980). Para determinar o Ki com maior precisão, esses autores sugerem que essa determinação seja feita por meio do teste de envelhecimento acelerado, com deterioração acelerada das sementes, sob condições constantes e adversas de umidade e temperatura (umidade de $16 \%$ e temperatura entre 40 e $45^{\circ} \mathrm{C}$ ), por determinado período de tempo. Testes de germinação sucessivos seriam realizados com amostras retiradas em intervalos regulares nas condições anteriormente descritas, calculando-se assim a curva de sobrevivência das sementes por meio da análise de probit (Finney, 1971). O valor de Ki poderia então ser determinado na interceptação da curva de sobrevivência das sementes, plotada em probit, no tempo zero de armazenamento (Equação 1).

É necessário conhecer o valor de $K i$, dentre outros parâmetros para utilização do aplicativo computacional SEEDSOLVE desenvolvido por Sinício (2007), na previsão das perdas de germinação e vigor de sementes armazenadas. A equação de Mauri et al. (2007) foi utilizada naquele aplicativo, preliminarmente, para calcular o valor de $\mathrm{Ki}$ em função dos resultados dos testes iniciais de germinação e envelhecimento acelerado. Entretanto, a equação foi desenvolvida apenas para soja e os dados experimentais foram obtidos para o teste de envelhecimento acelerado na temperatura de $41^{\circ} \mathrm{C}$ por 72 horas (ISTA, 1995), enquanto que nesta pesquisa, optou-se por utilizar temperatura de $42^{\circ} \mathrm{C}$ por 96 horas (AOSA, 1983; Marcos Filho, 1999). Além 
disso, para tornar o aplicativo SEEDSOLVE mais versátil, seria interessante poder calcular o valor de $K i$ em função do resultado dos testes iniciais de germinação e também de outros testes de vigor, usualmente empregados para milho e soja, além do envelhecimento acelerado.

Assim, considerando-se a necessidade de determinar a qualidade inicial de sementes de milho e soja para utilização no modelo de probit, objetivou-se equacionar Ki em função do resultado dos testes iniciais de germinação e vigor (envelhecimento acelerado para milho e soja, frio sem solo para milho e tetrazólio para soja).

\section{MATERIAL E MÉTODOS}

O trabalho foi conduzido no Laboratório de Pesquisa em Sementes do Departamento de Fitotecnia da Universidade Federal de Viçosa, no período de agosto a dezembro de 2007.

Foram utilizados onze lotes de sementes de diferentes cultivares de milho (um de BR-106, um de SHS-5070, três de SHS-3031, dois de SHS-4050 e quatro de SHS5050), fornecidos pela empresa Santa Helena Sementes, localizada em Sete Lagoas, MG, referentes ao ano agrícola de 2006/2007. Também foram utilizados dados da literatura de seis lotes do cultivar BR-106 publicados por Peixoto et al. (1998), de quatro lotes comerciais de um híbrido publicados por Ávila et al. (2007) e de cinco lotes de híbrido simples (HS-200) publicados por Andrade et al. (2001).

Para a soja, foram utilizados onze lotes de sementes de diferentes cultivares (três de Conquista, quatro de Favorita e quatro de Vencedora), fornecidos pela empresa Agromen Sementes Agrícolas, oriundos de campo de produção de sementes localizado em Pirapora, MG, referentes ao ano agrícola de 2006/2007. Também foram utilizados dados da literatura de quatro lotes do cultivar IAC-8 e de quatro lotes do cultivar IAC-15 publicados por Dias et al. (1996) e de oito lotes de diferentes cultivares (BRS-133, BRS-134, BRS-155, BRS-184, CD-210, Embrapa-48, M-SOY-5942, M-SOY-7501) publicados por Schuab et al. (2002).

Foram realizados os testes e determinações discriminados a seguir.

Grau de umidade: foi determinado pelo método da estufa a $105 \pm 3^{\circ} \mathrm{C}$, durante $24 \mathrm{~h}$, utilizando-se duas repetições, com aproximadamente $10 \mathrm{~g}$ de sementes, conforme Regras para Análise de Sementes (Brasil, 1992).

Germinação (TG): foi utilizado o substrato rolo de papel Germitest, umedecido com água destilada na proporção de
2,5 vezes o peso do papel seco, temperatura do germinador de $25^{\circ} \mathrm{C}$ e quatro subamostras de 50 sementes por repetição (Brasil, 1992).

Envelhecimento acelerado (TEA): foi conduzido utilizando-se três repetições de 200 sementes cada para cada lote. Adotou-se a metodologia recomendada pelo Comitê de Vigor da Association of Official Seed Analysis (AOSA, 1983) e complementada por Marcos Filho (1999). Uma camada única de sementes foi colocada sobre telas metálicas acopladas a caixas plásticas tipo gerbox contendo $40 \mathrm{~mL}$ de água ao fundo. As caixas tampadas foram mantidas em BOD, a $42^{\circ} \mathrm{C}$ durante 96 horas, para milho, e $41^{\circ} \mathrm{C}$ durante 48 horas, para soja. Decorridos esses períodos, quatro subamostras de 50 sementes de cada espécie, foram colocadas para germinar, conforme a metodologia descrita para germinação (Brasil, 1992). As avaliações foram realizadas aos quatro e cinco dias após a semeadura para milho e soja, respectivamente. Os resultados foram expressos em porcentagem de plântulas normais.

Frio sem solo (TF): foi conduzido para sementes de milho de maneira semelhante ao TG com as modificações que seguem. Após a confecção, os rolos de papel foram colocados em sacos de polietileno e em incubadora a $10^{\circ} \mathrm{C}$, durante sete dias; em seguida, os rolos foram transferidos para o germinador a $25^{\circ} \mathrm{C}$ e a avaliação foi realizada no quarto dia. Os resultados foram expressos em porcentagem de plântulas normais.

Tetrazólio (TZ): foi utilizado para avaliar o vigor (classes 1 a 3) dos lotes de sementes de soja. Foram empregadas 100 sementes por lote, distribuídas em quatro repetições de 25 sementes. Inicialmente as sementes foram pré-condicionadas entre folhas de papel-toalha umedecidas e levadas ao germinador, a $25^{\circ} \mathrm{C}$, durante $16 \mathrm{~h}$, aproximadamente. Após esse período, as sementes foram colocadas em recipientes de plástico (copos descartáveis de $50 \mathrm{~mL}$ ) e imersas em solução de sal 2,3,5 cloreto de trifenil-tetrazólio na concentração de $0,075 \%$, e mantidas a $35^{\circ} \mathrm{C}$, por período aproximado de 150 a 180min, quando apresentaram coloração adequada para serem avaliadas (França Neto et al., 1998). Em seqüência, as sementes foram lavadas em água corrente e mantidas submersas em água até o momento da avaliação. As sementes foram avaliadas individualmente, seccionandose-as longitudinalmente com o auxílio de uma lâmina de barbear, observando-se a ocorrência de danos (mecânico, secagem, beneficiamento) nas partes externas e internas dos cotilédones, dando atenção especial ao eixo embrionário (França Neto, 1994). Observou-se ainda, a diferenciação de cores dos tecidos, de acordo com os critérios estabelecidos por 
Moore (1985), ou seja, vermelho brilhante ou rosa brilhante (tecido vivo e vigoroso), vermelho carmim forte (tecido em deterioração) e branco leitoso ou amarelado (tecido morto). Os resultados foram expressos em porcentagem de sementes viáveis vigorosas.

\section{Determinação Experimental de $\mathbf{K i}$}

Foram conduzidos experimentos de armazenagem, em condições de grau de umidade e temperatura constantes, para os onze lotes de sementes de milho e de soja fornecidos pelas empresas; Santa Helena Sementes e Agromen Sementes Agrícolas, respectivamente, objetivando determinar o valor de Ki. Para isto, utilizou-se metodologia descrita por Ellis e Roberts (1980), com a diferença de que se optou por armazenar as sementes com o grau de umidade em que foram embaladas nas empresas para posterior comercialização. Optou-se por este procedimento porque em testes preliminares, verificouse que as condições de umidade, temperatura e período de armazenagem sugerida por Ellis e Roberts (1980), ocasionaram crescimento de fungos em algumas amostras de sementes, além da possibilidade de que o processo de umedecimento poderia causar danos fisiológicos às sementes. Assim, amostras contendo cerca de 400 sementes de milho com grau de umidade de $12,4 \pm 0,1 \%$ e de soja com $9,8 \pm 0,5 \%$, foram colocadas em embalagens de polietileno (impermeáveis à água), e depois foram armazenadas em uma incubadora B.O.D. à temperatura pré-ajustada de $47^{\circ} \mathrm{C}$ por 77 dias para o milho e de $45^{\circ} \mathrm{C}$ por 24 dias para a soja. A cada três dias, durante o período de armazenagem, foi retirada uma amostra de cada embalagem e realizou-se o teste de germinação (Brasil, 1992). Os resultados foram obtidos em percentagem de plântulas normais e transformados em probit. Posteriormente, esses resultados foram submetidos a análises estatísticas, obtendo-se a curva de sobrevivência das sementes e em seguida foi determinado o valor de Ki (ponto de interceptação do eixo Y) por meio de regressões lineares.

\section{Análises Estatísticas}

Os resultados da estimativa de $K i$, juntamente com o resultado dos testes iniciais de germinação e de envelhecimento acelerado para milho e soja, transformados em probit, foram submetidos a análises de regressão linear múltipla para equacionar a qualidade inicial das sementes em função do resultado desses testes. Os resultados dos testes de vigor, juntamente com os dados obtidos na literatura, foram submetidos a análises de regressão linear simples para equacionar o resultado dos testes envelhecimento acelerado em função do resultado dos testes de frio para milho e tetrazólio para soja.

\section{RESULTADOS E DISCUSSÃO}

Os resultados experimentais dos testes iniciais de umidade, germinação, vigor e das regressões lineares para determinar a qualidade inicial das sementes de diferentes cultivares de milho e soja, são apresentados nas Tabelas 1 e 2, respectivamente. As curvas de sobrevivência, experimentais e calculadas, obtidas na determinação do valor de $K i$, para diferentes cultivares de milho e soja, são mostradas na Figura 1. A determinação de $K i$ por meio de regressões lineares utilizando as curvas de sobrevivência resultou em valores médios do coeficiente de regressão ajustado $\left(\mathrm{r}^{2}\right)$ de 0,72 e 0,85 , para milho e soja, respectivamente (Tabelas 1 e 2 ). Os ajustes obtidos nessas regressões podem ser considerados relativamente bons tendo-se em conta os desvios padrões

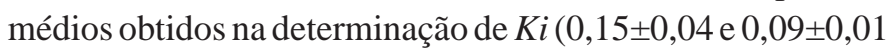
probit, para milho e soja, respectivamente).

Pieta Filho et al. (1992) obtiveram desvio padrão médio na determinação de $K i$ de $0,16 \pm 0,06$ probit (seis lotes de cevada e oito lotes de trigo) e Fabrizius et al. (1999) obtiveram $0,46 \pm 0,31$ probit (12 lotes de soja). Portanto, verifica-se que os desvios padrões médios de Ki obtidos na presente pesquisa, são menores do que os obtidos por aqueles pesquisadores.

Em alguns lotes de sementes de soja (Vencedoras 50007, 50071 e 50018, Favoritas 50109 e 50139) foi necessário eliminar os dados do período inicial de armazenamento (até 6 dias) porque as reduções de germinação não foram significativas neste período. $\mathrm{Na}$ maioria dos lotes de sementes de milho foi necessário eliminar dados do período final (mais de 47 dias) porque estes apresentaram aumento na porcentagem de plântulas anormais.

As análises de regressão linear múltipla para equacionamento de $K i$ em função de TG e TEA para milho e soja, utilizando os dados apresentados nas Tabelas 1 e 2 , resultaram na seguinte equação:

$K i=0,4230+0,4464 T G+0,4131$ TEA

A equação de $K i$ apresentou $r^{2}$ de 0,80 , desvio padrão de 0,18 probit e os parâmetros foram significantes em $\mathrm{p}<$ $0,01, p<0,02$ e $p<0,05$, respectivamente (total de 20 pontos experimentais). Os lotes SHS-5050 19M e Vencedora-50071 foram excluídos dessa análise porque a sua inclusão causou redução do $\mathrm{r}^{2}$ para 0,65 e aumento do desvio padrão para 0,24 probit. Por causa disso, esses lotes não foram incluídos nas Figuras 1 e 2. 
TABELA 1. Resultados experimentais dos testes iniciais de umidade, germinação (TG), envelhecimento acelerado (TEA), frio sem solo (TF) e das regressões lineares para determinar a qualidade inicial $\left(\mathrm{Ki},-1 / \sigma, \mathbf{r}^{2}-\right.$ ajustado, desvio padrão-d.p.) para sementes de diferentes cultivares de milho.

\begin{tabular}{|c|c|c|c|c|c|c|c|c|}
\hline \multirow[b]{2}{*}{ Lotes } & \multirow[b]{2}{*}{ Umidade (\%) } & \multirow[b]{2}{*}{ TG (\%) } & \multirow[b]{2}{*}{ TEA $(\%)$} & \multirow{2}{*}{$\mathrm{TF}(\%)$} & \multicolumn{4}{|c|}{ Regressões lineares } \\
\hline & & & & & $\begin{array}{c}K i \\
\text { (probit) }\end{array}$ & $\begin{array}{c}-1 / \sigma \\
\text { (probit) }\end{array}$ & $\mathrm{r}^{2}$ ajustado & $\begin{array}{c}\text { d.p. } \\
\text { (probit) }\end{array}$ \\
\hline BR-106 19L & 12,3 & 93,5 & 83,0 & 91,0 & 1,32 & $-0,01241$ & 0,87 & 0,12 \\
\hline SHS-3031 19L & 12,4 & 91,0 & 74,0 & 88,0 & 1,37 & $-0,01402$ & 0,57 & 0,17 \\
\hline SHS-3031 20L & 12,3 & 89,5 & 84,0 & 90,5 & 1,39 & $-0,01416$ & 0,74 & 0,20 \\
\hline SHS-3031 20M & 12,5 & 88,5 & 88,0 & 91,0 & 1,38 & $-0,00820$ & 0,70 & 0,12 \\
\hline SHS-4050 18RL & 12,0 & 91,0 & 92,0 & 91,0 & 1,57 & $-0,02764$ & 0,89 & 0,08 \\
\hline SHS-4050 24RL & 12,3 & 92,0 & 93,5 & 91,0 & 1,48 & $-0,00477$ & 0,59 & 0,10 \\
\hline SHS-5050 18M & 12,2 & 89,0 & 84,5 & 95,0 & 1,59 & $-0,02893$ & 0,55 & 0,20 \\
\hline SHS-5050 18R & 12,0 & 90,0 & 88,5 & 90,5 & 1,34 & $-0,02048$ & 0,93 & 0,08 \\
\hline SHS-5050 19M & 12,0 & 94,5 & 90,0 & 88,5 & 1,12 & $-0,01074$ & 0,65 & 0,11 \\
\hline SHS-5050 22C & 12,3 & 89,0 & 93,5 & 91,0 & 1,63 & $-0,02602$ & 0,78 & 0,20 \\
\hline SHS-5070 18C & 11,6 & 91,5 & 91,0 & 90,5 & 1,79 & $-0,01998$ & 0,67 & 0,20 \\
\hline Média & 12,4 & 92,3 & 78,5 & 89,5 & 1,35 & $-0,01322$ & 0,72 & 0,15 \\
\hline Desvio padrão & 0,1 & 1,8 & 6,4 & 2,1 & 0,04 & 0,00114 & 0,21 & 0,04 \\
\hline
\end{tabular}

TABELA 2. Resultados experimentais dos testes iniciais de umidade, germinação (TG), envelhecimento acelerado (TEA), tetrazólio (TZ) e das regressões lineares para determinar a qualidade inicial (Ki, -1/ $\sigma, \mathbf{r}^{2}$-ajustado, desvio padrão-d.p.) para sementes de diferentes cultivares de soja.

\begin{tabular}{lcccccccc}
\hline & & & & & \multicolumn{3}{c}{ Regressões lineares } \\
\cline { 7 - 9 } \multicolumn{1}{c}{ Lotes } & $(\%)$ & $(\%)$ & $(\%)$ & $(\%)$ & $K i$ & $-1 / \sigma$ & $\mathrm{r}^{2}$ ajustado & $\begin{array}{c}\text { d.p. } \\
\text { (probit) }\end{array}$ \\
\hline Conquista-50210 & 9,4 & 88 & 86 & 80 & 1,15 & $-0,03277$ & 0,90 & 0,08 \\
Conquista-50238 & 10,1 & 71 & 75 & 68 & 1,04 & $-0,03180$ & 0,79 & 0,09 \\
Conquista-50239 & 10,8 & 81 & 67 & 70 & 1,07 & $-0,05516$ & 0,93 & 0,12 \\
Favorita-50109 & 10,3 & 81 & 71 & 57 & 1,50 & $-0,06513$ & 0,68 & 0,24 \\
Favorita-50115 & 9,6 & 82 & 63 & 56 & 0,97 & $-0,03874$ & 0,70 & 0,19 \\
Favorita-50139 & 9,5 & 63 & 46 & 41 & 0,61 & $-0,04128$ & 0,93 & 0,12 \\
Favorita-50143 & 9,6 & 57 & 45 & 44 & 0,17 & $-0,02554$ & 0,91 & 0,06 \\
Vencedora-50007 & 10,3 & 86 & 85 & 73 & 1,33 & $-0,03168$ & 0,81 & 0,09 \\
Vencedora-50018 & 9,6 & 82 & 88 & 69 & 1,40 & $-0,03382$ & 0,71 & 0,10 \\
Vencedora-50070 & 9,3 & 68 & 44 & 63 & 0,44 & $-0,03706$ & 0,91 & 0,10 \\
Vencedora-50071 & 9,6 & 62 & 34 & 35 & 0,96 & $-0,06559$ & 0,60 & 0,24 \\
Média & 9,8 & 79,5 & 80,5 & 74,0 & 1,10 & $-0,03229$ & 0,85 & 0,09 \\
Desvio padrão & 0,5 & 12,0 & 7,8 & 8,5 & 0,08 & 0,00069 & 0,08 & 0,01 \\
\hline
\end{tabular}



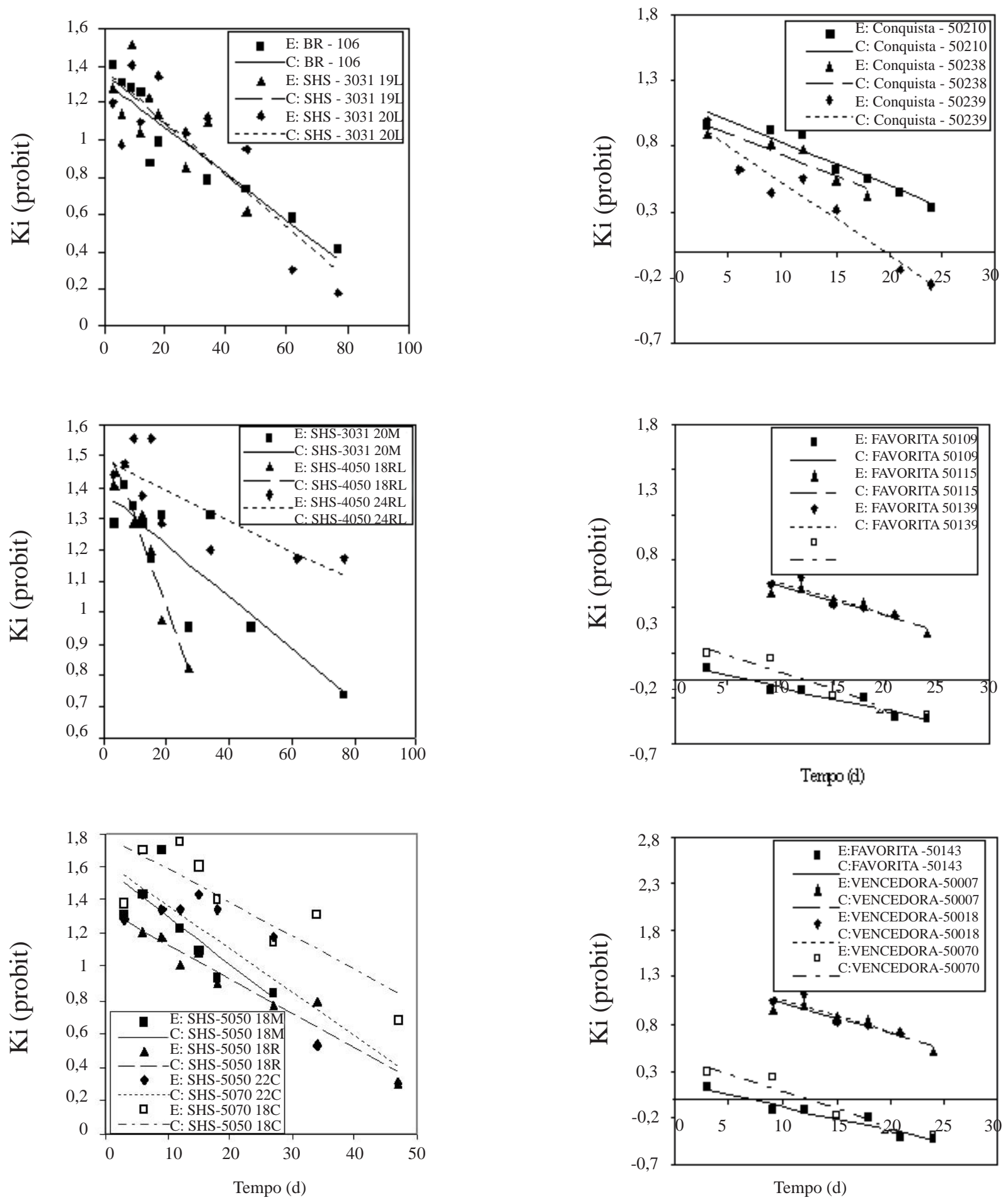

FIGURA 1. Curvas de sobrevivência, experimentais e calculadas, obtidas na determinação do valor de $\mathrm{Ki}$, para sementes de diferentes cultivares de milho (a) e soja (b).

Observa-se, graficamente, que a equação de $K i$ se ajustou relativamente bem aos resultados dos testes iniciais de germinação e envelhecimento acelerado, tanto para milho como para soja (Figura 2). Os valores da qualidade inicial calculados pela equação de Mauri et al. (2007) apresentaram $\mathrm{r}^{2}$ de 0,77 e desvio padrão de 0,21 quando comparados aos 
valores experimentais de Ki mostrados nas Tabelas 1 e 2 . A comparação gráfica dos resultados experimentais de Ki desta pesquisa com aqueles calculados por Mauri et al. (2007) também mostram ajuste relativamente bom (Figura 2). Os resultados da presente pesquisa, portanto, confirmam os resultados obtidos por estes autores, apesar das diferenças existentes na metodologia utilizada para determinação de Ki.

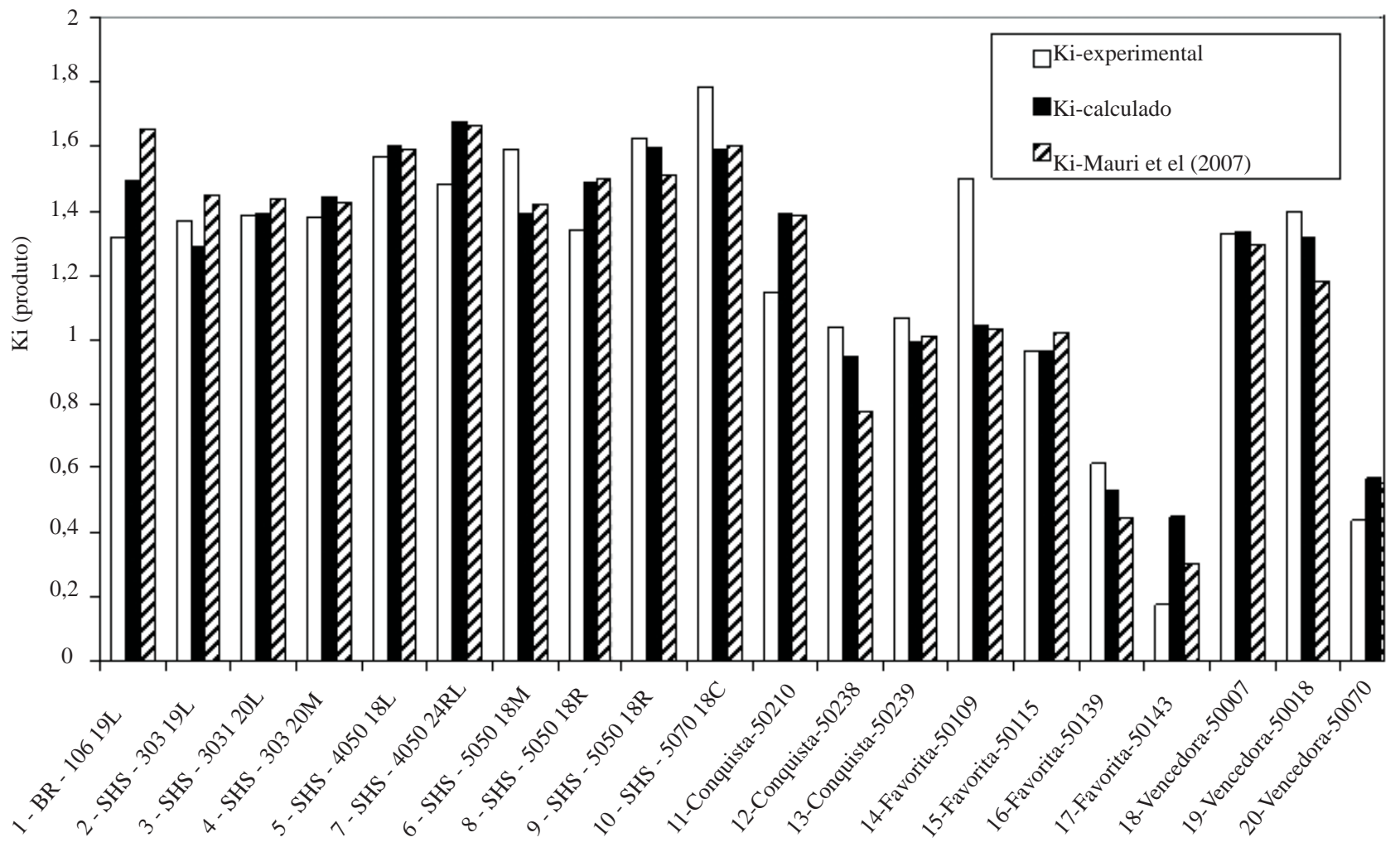

FIGURA 2. Valores experimentais e calculados da qualidade inicial Ki para lotes de sementes de milho e soja em função do resultado dos testes iniciais de germinação e envelhecimento acelerado.

As análises de regressão linear múltipla para equacionamento de $K i$ em função de $T G$ e $T F$ ou $T G$ e $T Z$ não produziram resultados satisfatórios, provavelmente, devido ao pequeno número de lotes de sementes de milho e soja utilizados. Desta forma, buscou-se equacionar, alternativamente, TEA em função de TF para milho e TEA em função de $T Z$ para soja, utilizando além dos dados apresentados nas Tabelas 1 e 2, dados publicados na literatura para milho (Peixoto et al., 1998; Ávila et al., 2007; Andrade et al., 2001) e soja (Dias et al., 1996; Schuab et al., 2002).

Análises de regressão linear resultaram em equações que estabelecem o valor de envelhecimento acelerado em função do teste de frio para milho (TEA $A_{m}$ ) e em função do teste de tetrazólio para soja $\left(T E A_{s}\right)$, conforme seguem:

$$
\begin{aligned}
& T E A_{M}=-0,4408+1,214 T F \\
& T E A_{S}=-0,1872+0,9875 T Z
\end{aligned}
$$

A equação de $T E A_{m}$ apresentou $r^{2}$ de 0,80 , desvio padrão de 0,24 probit e os parâmetros foram significantes em $p$ $<0,002$ (total de 25 pontos experimentais). A equação de $T E A_{s}$ apresentou $\mathrm{r}^{2}$ de 0,84 , desvio padrão de 0,18 probit e os parâmetros foram significantes em $\mathrm{p}<0,005$ (total de 25 pontos experimentais). Valores experimentais e calculados do resultado do teste de envelhecimento acelerado de sementes de diferentes cultivares, plotados em função do resultado do teste de frio sem solo para milho e do resultado do teste de tetrazólio para soja, podem ser observados nas Figuras 3 e 4, respectivamente. 


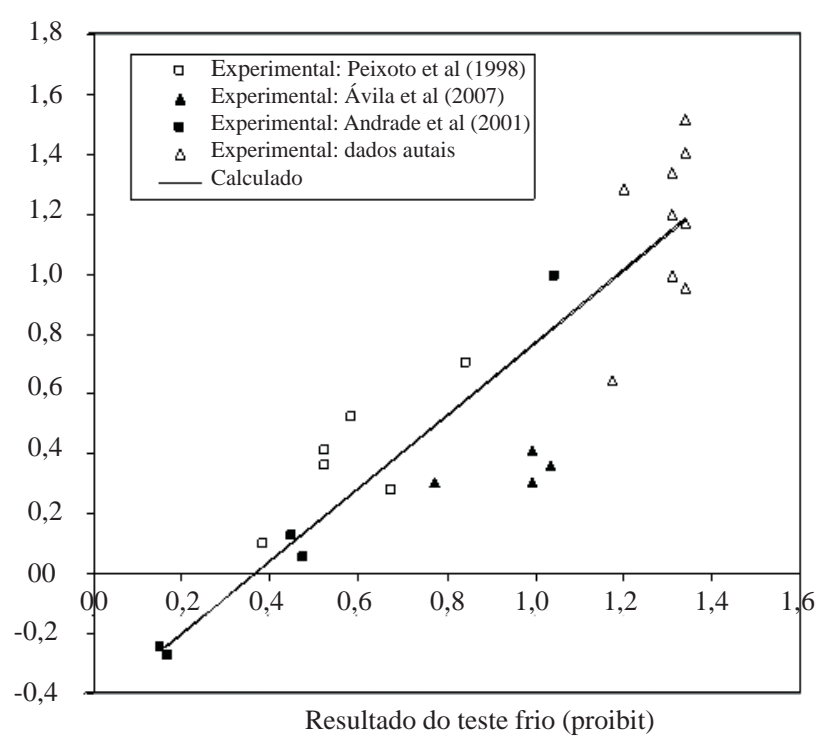

FIGURA 3. Valores experimentais e calculados do resultado do teste de envelhecimento acelerado de sementes de diferentes cultivares de milho em função do resultado do teste de frio sem solo.

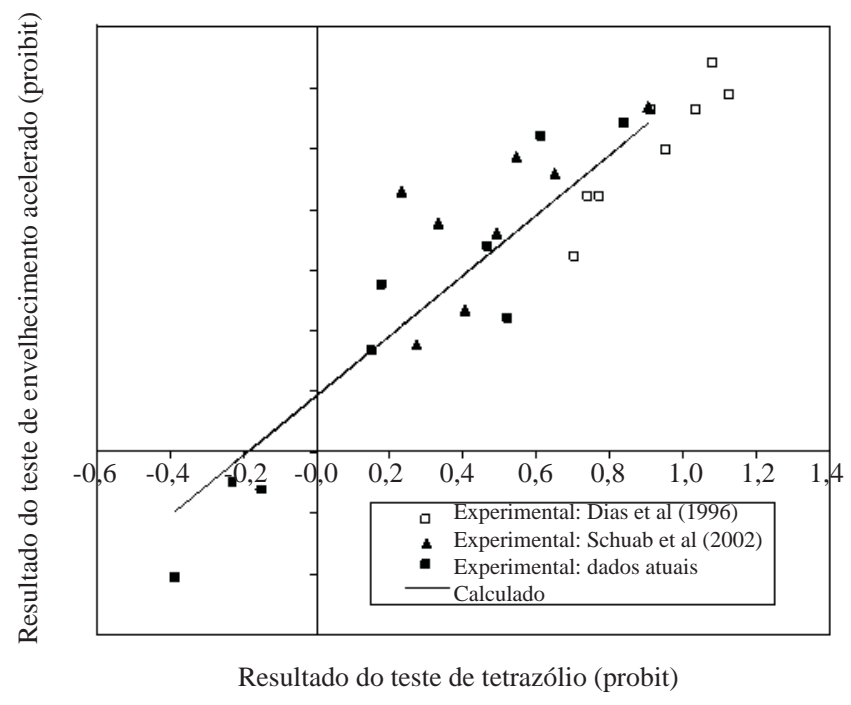

FIGURA 4. Valores experimentais e calculados do resultado do teste de envelhecimento acelerado de sementes de diferentes cultivares de soja em função do resultado do teste de tetrazólio.

O lote SHS-5050 18M foi excluído do equacionamento de $T E A_{m}$ porque a sua inclusão causou redução do $\mathrm{r}^{2}$ para 0,77 e aumento do desvio padrão para 0,26 probit. Também, os lotes Vencedora-50018 e 50070 foram excluídos do equacionamento de $T E A_{\text {s }}$ porque a sua inclusão causou redução do $r^{2}$ para 0,74 e aumento do desvio padrão para 0,24 probit. Todos os dados publicados por Ávila et al. (2007) foram utilizados com exceção de um lote de milho híbrido com TEA de 34\% e todos os dados publicados por Schuab et al. (2002) foram utilizados com exceção de dois lotes de soja com TEA menores ou iguais a $28 \%$.

\section{CONCLUSÕES}

A estimativa da qualidade inicial das sementes pode ser determinada em função do resultado dos testes iniciais de germinação e envelhecimento acelerado para sementes de milho e soja;

$\mathrm{O}$ resultado do teste de envelhecimento acelerado para milho pode ser determinado em função do resultado do teste de frio sem solo;

O resultado do teste de envelhecimento acelerado para soja pode ser estabelecido em função do resultado do teste de tetrazólio $(T Z)$.

\section{REFERÊNCIAS}

ANDRADE, R.V.; AUZZA, S.A.Z.; ANDREOLI, C.; NETTO, D.A.M.; OLIVEIRA, A.C. Qualidade fisiológica das sementes do milho híbrido simples HS 200 em relação ao tamanho. Ciência Agrotécnica, Lavras, v.25, n.3, p.576582, 2001.

ASSOCIATION OF OFFICIAL SEED ANALYSTS. Seed Vigor Test Committee. Seed Vigor Testing Handbook. Lincoln, 1983. 88p. (Contribution, 32).

ÁVILA, M.R.; BRACCINI, A.L.; SCAPIM, C.A. Teste de comprimento de plântulas sob estresse hídrico na avaliação do potencial fisiológico das sementes de milho. Revista Brasileira de Sementes, Londrina, v.29, n.2, p.117-124, 2007.

BEWLEY, J.; BLACK, M. Seeds. Physiology of development and germination, Plenum Press, New York, 1994.

BRASIL. Ministério da Agricultura e Reforma Agrária. Secretaria Nacional de Defesa Agropecuária. Regras para análise de sementes. Brasília: 1992. 365p.

DIAS, D.C.F.S.; MARCOS-FILHO, J.; CARMELLO, Q.A.C. Potassium leakage test for the evaluation of vigour in soybean seeds. Seed Science and Technology, v.25, p.7$18,1996$.

ELLIS, R. H.; ROBERTS, E. H. Improved equations for the 
prediction of seed. longevity. Annals of Botany, v.45, p.1330, 1980.

FABRIZIUS, E.; TEKRONY, D.; EGLI, D. B.; RUCKER, M. Evaluation of a viability model for predicting soybean seed germination during warehouse storage. Crop Science. v.39, p.194-201, 1999.

FINNEY, D.J. Probit analysis. London: Cambridge University Press, 1971. 333p.

FRANÇA NETO, J.B. O teste tetrazólio em Sementes de Soja. IN: VIEIRA, R.D., CARVALHO, N.M. ed. Testes de vigor em sementes. Jaboticabal, FUNEP/UNESP, 1994. p.87-102.

FRANÇA NETO, J.B. FRANÇA NETO, J.B. KRZYZANOWSKI, F.C.; COSTA, N.P. da. O teste de tetrazólio em sementes de soja. Londrina: EMBRAPACNPSo, 1998. 72p. (EMBRAPA-CNPSo, Documentos, 116).

HONG, T.D., LININGTON, S., ELLIS, R.H. Seed Storage Behaviour: a Compendium. Handbooks for Genebanks: No. 4. International Plant Genetic Resources Institute, Rome, 1996. 120p.

International Seed Testing Association. Handbook of vigour test methods. ISTA, Zurich, Switzerland, 1995.

MARCOS FILHO, J. Teste de envelhecimento acelerado. In: KRZYZANOWSKI, F.C.; VIEIRA, R.D.; FRANÇA NETO, J.B. (Ed.) .Vigor de Sementes: conceitos e testes. Londrina: ABRATES, 1999. cap.3, p.1-24.

MAURI, A. L.; ARAUJO, E.F.; SINÍCIO, R.; REIS,
M.S.; DIAS, D.C.F.S; CAMPOS, J.A. Equacionamento da qualidade inicial de semente de soja no modelo de probit. Revista Brasileira de Armazenamento,Viçosa, v.32, n.1, p.14-22, 2007.

MEAD, A.; GRAY, D. Prediction of seed longevity: a modification of the shape of the Ellis and Roberts seed survival curves. Seed Science Research, v.9, p.63-73, 1999.

MOORE, R.P. Handbook on tetrazolium testing. Zurich: ISTA, 1985, 99p.

PEIXOTO, A.R.; TORRES, S.B.; KARASAWA, M. Qualidade sanitária e fisiológica de sementes de milho produzidas no submédio São Francisco. Revista Brasileira de Sementes, Londrina, v.20, n.1, p.12-15, 1998.

PIETA FILHO, C.; ELLIS, R.H. Estimating the value of the seed lot Constant (Ki)of the seed viability equation in barley and wheat. Seed Science and Technology, v.20, p.93-99, 1992.

SCHUAB, S.R.P.; BRACCINI, A.L.; FRANÇA NETO, J.B.; SCAPIM, C.A.; MESCHEDE, D.K. Utilização da taxa de crescimento as plântulas na avaliação do vigor de sementes de soja. Revista Brasileira de Sementes, Londrina, vol. 24, n.2, p.90-95, 2002.

SINÍCIO, R. Desenvolvimento do aplicativo computacional SEEDSOLVE para previsão das perdas de germinação e vigor de sementes armazenadas. Trabalho submetido para publicação na Revista Brasileira de Sementes, Londrina, Protocolo n.125/2007, 2007. 